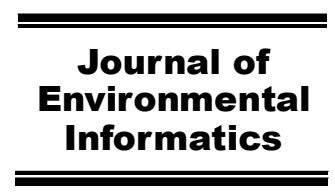

www.iseis.org/jei

\title{
Kalman Filter for Subsurface Transport Models with Inaccurate Parameters and Unknown Sources
}

\author{
A. Jin and S. Y. Chang ${ }^{*}$ \\ Civil \& Environmental Engineering, North Carolina A\&T State Univ., Greensboro NC 27411, USA
}

Received 5 December 2007; revised 19 March 2008; accepted 27 June 2008; published online 2 September 2008

\begin{abstract}
In order to improve the contaminant plume prediction in subsurface transport models, a data assimilation scheme using the Kalman filter (KF) is developed. The data assimilation scheme is designed to reduce uncertainties in model predictions. These uncertainties actually represent all the unpredictable variations due to the unknown or uncertain properties in physical law based models and the incomplete knowledge of stochastic fields. Considering the background of subsurface transport is spatially dependent, simulation of spatially correlated uncertainties are proposed and integrated into a data assimilation scheme. Sequential instances of spatially correlated random fields are simulated in examining the effectiveness of the assimilation system. Results show that this data assimilation scheme reduces the uncertainty of predictions from the deterministic model. By assimilating the observations, the predictive contaminant plumes from the assimilation system can trace the randomized irregular plume shape of the assumed case more closely than a non-assimilation deterministic model. The statistical representation of random noise, such as spatially dependency, is critical in improving effectiveness of the statistical optimization process. In two test cases which simulate the scenarios with unknown hidden sources or inaccurate hydrologic properties, the regional noise KF scheme demonstrates the potentials to solve the inverse problems in environmental transport modeling.
\end{abstract}

Keywords: subsurface transport, uncertainty, inverse problem, Kalman filter, data assimilation, spatial variation

\section{Introduction}

Subsurface properties vary in space and time. In practices, finite properties of the subsurface can be obtained by measuring them in a limited number of sites during a limited sampling period. However, the knowledge of the properties can never be considered complete as required by deterministic models that are governed by well-established physical laws. In general, the prediction is inevitably uncertain (Heuvelink and Webster, 2001) and some random variables must be introduced to represent the unpredictable variation. Therefore, stochastic data assimilation such as Kalman filter (KF) can be integrated with deterministic models to give better predictions.

Many studies (Ghil et al., 1981; Gustafsson, 1981; Houtekamer and Mitchell, 1998) proposed that the KF (Kalman and Bucy, 1961) in sequential estimation theory could be applied to meteorological data assimilation. KF was also used in areas of surface and subsurface hydrologic systems to obtain the optimal estimation (Bowles and Greney, 1978; Van Geer, 1982; Yu et al., 1989; Yangxiao et al., 1991; Ferraresi and Marinelli, 1996; Harrouni et al., 1997; Porter et al., 2000). As

${ }^{*}$ Corresponding author. Tel.: +1 336 3347737; fax: +1 3363347126.

E-mail address: chang@ncat.edu (S. Y. Chang).

ISSN: $1726-2135$ print/1684-8799 online

(C) 2008 ISEIS All rights reserved. doi:10.3808/jei.200800122 pointed by Porter et al. (2000), due to the spatial physical and statistical relationship of groundwater system, KF should treat the computations over space differently from it does over time.

Statistical representation of this variation of subsurface properties is critical for improving effectiveness of the statistical optimization process. Even though this uncertainty cannot be avoided, its spatial and temporal variation pattern can be simulated and integrated into the prediction models. Actually, the quality of KF greatly depends on the noise structures for the measurements and the system. Spatially independent Gaussian noise is easy to obtain and is widely assumed in many areas for examining KF properties. For subsurface modeling, however, it is more reasonable to consider the data and noise as regional i.e. spatially correlated instead of spatially independent Gaussian (Webster and Oliver, 1992; Conwell et al., 1997; Goovaerts, 1999; Brooker, 2001; Ferreyra et al., 2002). In this study, a KF is constructed as a data assimilation scheme for a subsurface contaminant model. Then a spatially random field simulation scheme that can be integrated into a $\mathrm{KF}$ is introduced to examine two specific cases. One is the prediction with inaccurate hydrologic parameters and the other is the identification of unknown contaminant sources.

\section{Methodology}

This system includes a deterministic physical transport model, a KF data assimilation scheme which can be integrated 
into the transport model, the numerical schemes for models and filter, non-Gaussian noise simulators and a test frame (Chang and Jin, 2005). For clarity, we describe briefly the core of the system as follows.

\subsection{Stochastic System}

In order to model transport processes which usually have uncertain sources, inaccurate transport parameters or numeric errors, the stochastic dynamics can be represented as deterministic dynamics of transport and a random noise term:

$\mathrm{x}_{k+1}=\mathrm{Ax}_{k}+\mathrm{p}_{k}(k=0,1,2,3, \ldots)$

where $\mathrm{x}_{k}$ and $\mathrm{x}_{k+1}$ are the state variables at time $k$ and $k+1$, respectively; $\mathrm{A}$ is the finite-difference operator that advances the $\mathrm{x}_{k}$ by one time step; $\mathrm{p}_{k}$ is the model system error; the difference between the model prediction and the optimal estimate of the true value. The model error vector $\left(\mathrm{p}_{k}\right)$ is assumed to have covariance matrix $\mathrm{Q}_{k}$ and zero mean.

Observation $\mathrm{z}_{k}$, which could be irregularly distributed in space at time scale, is represented by data conversion matrix $\mathrm{H}$ and random errors $\mathrm{o}_{k} . \mathrm{O}_{k}$ is assumed to be the error of observations that has covariance matrix $\mathrm{R}_{k}$ and zero mean:

$\mathrm{z}_{k}=\mathrm{Hx}^{T}{ }_{k}+\mathrm{o}_{k}$

\subsection{Optimal Estimation and Data Assimilation with KF}

The Kalman optimal estimator is expressed as:

$\mathrm{x}_{k+1}(+)=\mathrm{x}_{k+1}(-)+\mathrm{K}_{k+1}\left[\mathrm{Z}_{k+1}-\mathrm{H} \mathrm{x}_{k+1}(-)\right]$

where $\mathrm{x}_{k+1}(+)$ indicates the estimated value after the KF adjustment, and $\mathrm{x}_{k+1}(-)$ the value before the $\mathrm{KF}$ adjustment, i.e. the predicted value from the model. The matrix $\mathrm{K}_{k+1}$ is determined by:

$\mathrm{K}_{k+1}=\mathrm{P}_{k+1}(-) \mathrm{H}^{\mathrm{T}}\left[\mathrm{H} \mathrm{P}_{k+1}(-) \mathrm{H}^{\mathrm{T}}+\mathrm{R}_{k+1}\right]^{-1}$

where $\mathrm{P}_{k+1}$ is the optimal estimate error covariance matrix, $\mathrm{M}^{\mathrm{T}}$ and $\mathrm{M}^{-1}$ denote the transpose and inverse of matrix $\mathrm{M}$, respectively. $\mathrm{P}_{k+1}$ can be obtained by recursive calculation:

$\mathrm{P}_{k+1}(+)=\mathrm{P}_{k+1}(-)-\mathrm{P}_{k+1}(-) \mathrm{H}^{\mathrm{T}}\left[\mathrm{HP}_{k+1}(-) \mathrm{H}^{\mathrm{T}}+\mathrm{R}\right]^{-1} \mathrm{HP}_{k+1}(-)$

and

$\mathrm{P}_{\mathrm{k}+1}(-)=\mathrm{AP}_{k}(+) \mathrm{A}^{\mathrm{T}}+\mathrm{Q}_{k}$

where $\mathrm{K}_{k+1}$ in Equation (4) is called the Kalman optimal gain matrix or Kalman filter. In addition to advancing a time sequence, at the same time, system operator A also applies to a space sequence, $x[i], i=0,1,2, \ldots$, where index $i$ indicates the space positions. Therefore, the KF has the potential for assi- milating data in both space and time. KF can be conveniently implemented since the model dynamic system itself, Equation (1), has already been integrated in the assimilation.

\subsection{Spatial Dependence and Spatial Random Noise Simu- lation}

Spatial dependence is the inherent nature of most geographically related data. Obviously, the properties of the variables in subsurface modeling belong to this category. However, the major methodologies that have been used to solve the subsurface transport processes, such as the numerical algorithms of differential equations and KF, are aspatial or statistical. Applying spatial dependence into subsurface model allows discovering of the spatial structure of the noise variables and improving accuracy of the simulations.

In geographic systems, spatial dependency is usually expressed by semi-variogram. Assume we have $\{Z(t): t \in T\}$, where $T \subseteq \mathfrak{R}^{d}$. Here $T$ is the spatial location index set. Z(.) represents the stochastic spatial process. $\{z(t): t \in T\}$ is used to express an instance of the $\mathrm{Z}($.$) . Semi-variogram \gamma$ is defined as:

$\gamma\left(t_{1}-t_{2}\right)=\frac{1}{2} \operatorname{var}\left[Z\left(t_{1}\right)-Z\left(t_{2}\right)\right]=\frac{1}{2} E\left\{\left[Z\left(t_{1}\right)-Z\left(t_{2}\right)\right]^{2}\right\}, \forall t \in T$

If $E\{Z(t)\}=m, \forall t \in T$ and $\gamma(\cdot)$ exists, $Z(\cdot)$ is called intrinsically stationary.

In a true field situation, Equation (8) could be used to obtain the experimental semi-variogram. Given the two locations $t$ and $(t+h)$, a measure of one-half of the mean square differences between the values $\mathrm{z}(t+h)$ and $\mathrm{z}(t)$ gives the semi-variogram. Here $h$ (known as the lag) is the inter-sample distance. If the conditions specified by the intrinsic hypothesis are met, the semi-variance can be estimated from sample data by:

$\gamma(h)=\frac{1}{2 n} \sum_{n}[z(t)-z(t+h)]^{2}$

Once being determined from the field observations, the semi-variogram parameters, in turn, can be used in design of regional noise scheme for both system and observation errors.

\subsection{Regional Noise Field Simulation}

For the state-space estimate problem, system noise is usually denoted as a vector $\mathrm{p}_{\mathrm{k}}$ where $\mathrm{p}_{\mathrm{k}}$ is $\mathrm{q}-\mathrm{by}-1$ column vector with covariance matrix Q. The statistical structure of Gaussian noise with time independence is usually described as:

$E\left\{p_{k}\right\}=0$ 
$E\left\{p_{k} p_{l}{ }^{\prime}\right\}=Q_{r} \delta_{k l}\left(\delta_{k l}=0\right.$ if $k \neq l, \delta_{k l}=1$ if $\left.k=l\right)$

where Q is q-by-q symmetric and positive semi-definite. There are several ways to introduce the spatial dependence into the noise field. Since the noise will be integrated into KF, the most convenient way is to create the noise that satisfies given error covariance structures or given semi-variogram structures.

Let $\mathrm{p}_{\mathrm{k}}(t)$ be a 2-D random noise state vector, $t \in G_{s} \subseteq \mathfrak{R}^{2}$, with given covariance matrix $\mathrm{Q}(\mathrm{h})$ that represents a certain spatial dependence of the $p_{k}(t)$ :

$Q_{i j}=Q\left(t_{j}-t_{i}\right)=Q\left(h_{i j}\right)$

where $t$ is space location index, $\mathrm{G}_{\mathrm{s}}$ is grid of system noise injection, $h_{i j}$ is distance between the space location $t_{i}$ and $t_{j}$.

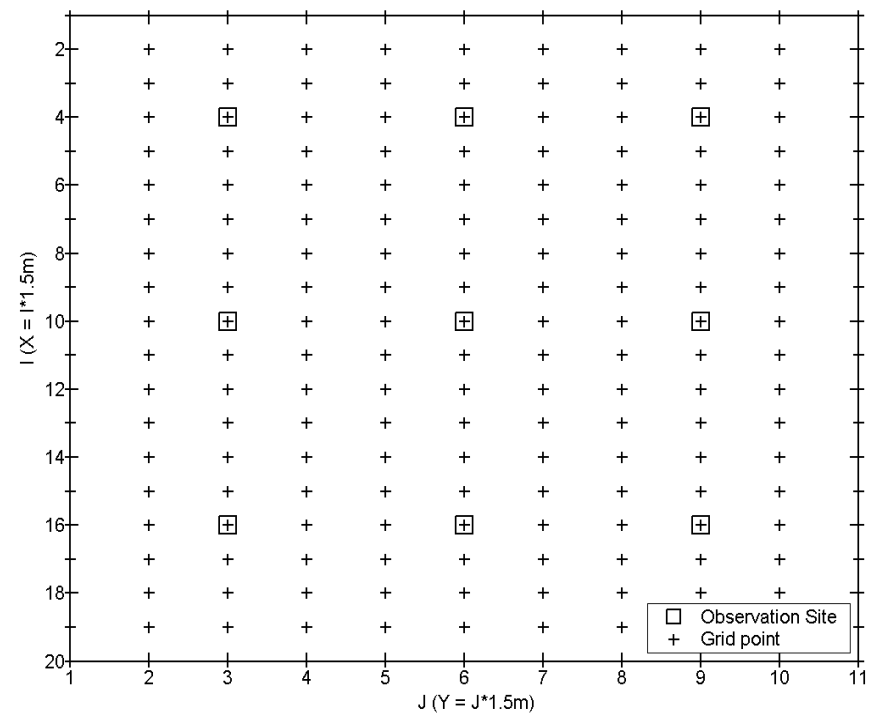

Figure 1. Numeric model grid and observation locations.

Assuming that the spatially-dependent $\mathrm{p}_{k}(t)$ has a reasonable decomposition with components of a spatially-independent unit Gaussian vector $\mathrm{g}_{\mathrm{k}}(\mathrm{t})$ and a linear transformation $\mathrm{L}$ :

$\mathrm{p}_{k}(t)=\operatorname{Lg}_{k}(t)$

with $E\left(\operatorname{gg}^{T}\right)=\mathrm{I}$, the linear transformation matrix L can be solved as the lower triangular of Cholesky decomposition of $\mathrm{Q}$ if $\mathrm{Q}$ is positive definite, i.e.

$\mathrm{Q}=\mathrm{LL}^{T}$

Basic spatially dependent noise can be used in various noise models to simulate more complicated error structures. This noise simulation scheme is applicable to observation err- or simulations.

\section{Experiments}

A two-dimensional numerical scheme is constructed in the horizontal plane ( $x-y$ plane) for simulating the contaminant advection-dispersion-adsorption process in a subsurface transport system:

$\frac{\partial C}{\partial t}=\frac{D_{x}}{R}\left(\frac{\partial^{2} C}{\partial x^{2}}\right)+\frac{D_{y}}{R}\left(\frac{\partial^{2} C}{\partial y^{2}}\right)-\frac{V}{R}\left(\frac{\partial C}{\partial x}\right)$

where $C$ is the concentration (mass of solute per unit mass of solution), $D_{x}$ and $D_{y}$ are the longitudinal and transverse dispersion coefficients, respectively, $R$ is the retardation factor, and $V$ is the average linear pore liquid velocity.

An initial spontaneous point mass source and a constant boundary condition were assumed:

$\left.C(x, y, t)\right|_{t=o}=C_{I}\left(\mathrm{x}_{0}, \mathrm{y}_{0}\right)$

and

$\left.C(x, y, t)\right|_{\Omega}=C_{B}$

where $\Omega$ is chosen as a square boundary and $C_{B}$ is the boundary value.

The model grid $\mathrm{G}_{\mathrm{m}}$ is defined on a 2-D plane domain. The pollution scenario is assumed as an instantaneous contaminant source injected into a location with coordinates $(5,6)$ in test case $1,(5,5)$ in test case 2 , with contamination background at $20 \mathrm{ppm}$. The details for setting domain (Figure 1) and other hydrologic parameters can be found in reference (Chang and Jin, 2005).

The simulated "true" data for the assumed pollution at the end of the time step are used to compare with the KF results. The purpose of this experiment is to demonstrate the efficiency of the regional noise that is used in KF assimilation. To eliminate the uncontrolled error directly introduced by the numerical frame, "true" data is simulated based on the same numerical model used in the prediction. Using this design, the system error $p_{k}$ can be completely under control and KF quality will therefore only show the impact of error structures that it relies on. Note in the experiments, true field is assumed as a realization of a stochastic process and used only in evaluating the impact of KF and regional field noise. That is, the prediction model and KF are not supposed to have knowledge of the "true" fields except through the limited observation sites. The true field has no fixed pattern and its contour shape varies from time to time for each different run due to the designed random noise scheme.

To implement the KF filter in the subsurface transport model, the model noise covariance matrix $\mathrm{Q}$ must be determined before operation of the filter. $\mathrm{Q}$ is the covariance ma- 
trix of the model error vector which is calculated based on the difference between the prediction of system model and the true solution at each cell. Q represents the inaccuracy of the prediction by the system model. It should also be pointed out that by simply injecting enough system error through the selection of $\mathrm{Q}_{\mathrm{k}}$ we could simulate the uncertainties, unknowns and incomplete knowledge of the transport process that results in system prediction error. In reality, since the true solution cannot be obtained, Q could usually be determined from the estimated model error by statistical or numerical analysis. In our testing cases, the reality is represented as different realizations of the stochastic process. Also, since the transport process and its predictions are actually occurring in a geographically specific subsurface area, it is obvious that to utilize a regional error frame to simulate the uncertainty is more reasonable than to use a geo-independent error frame. Therefore, a time-independent regional Q based on the Gaussian error with $\sigma_{s y s}=8$ ppm (Conwell et al., 1997; Webster and Oliver, 1992) were specified in test.

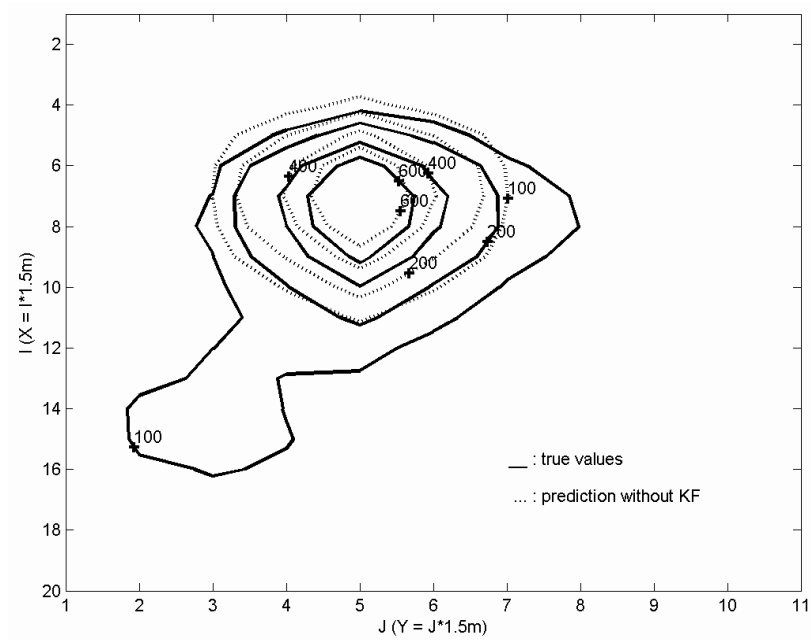

Figure 2. Prediction without KF data assimilation.

\section{Results and Discussions}

\subsection{Test Case 1 - Prediction with Inaccurate Hydrologic Parameters}

Although finite knowledge of hydrodynamic process in subsurface can be obtained by measuring them in the specified places at designed sampling times, the sampling places and times are restricted in practice. Description of the hydrologic parameters can never be considered complete and accurate as required by subsurface transport models. More effort in continuously pursuing completeness and accuracy in the labs and sites may reduce the errors caused by these hydrological parameters, but this is usually very costly due to highly heterogeneous subsurface environment. Applying KF data assimilation scheme can provide another cost-effective way to improve accuracy by merging finite observations.

In this experiment, we simulate the scenario that has one inaccurate hydrologic parameter in the prediction model and test the impact of regional noise R. For simplicity, the average linear pore liquid velocity, $\mathrm{V}$, is assumed inaccurate in the prediction model. $\mathrm{V}$ is increased by $20 \%$ when the true fields were simulated. However, a deterministic model without the correct input still employs the given $\mathrm{V}$ in its prediction.

To test the impact of error covariance structure on the correction process of the $\mathrm{KF}$ scheme, a $2 \mathrm{D}$ regional observation noise field (Chang and Jin, 2005) is created according to the specified covariance matrix $\mathrm{R}$ and applied to the data assimilation scheme in this experiment. The characteristics of regional noise can also be clearly shown from its covariance matrix. Compared to SIG R, the observation errors' statistical structure has been given a geospatial nature, i.e. at closer observation sites errors tend to be more covariant. Actually, in
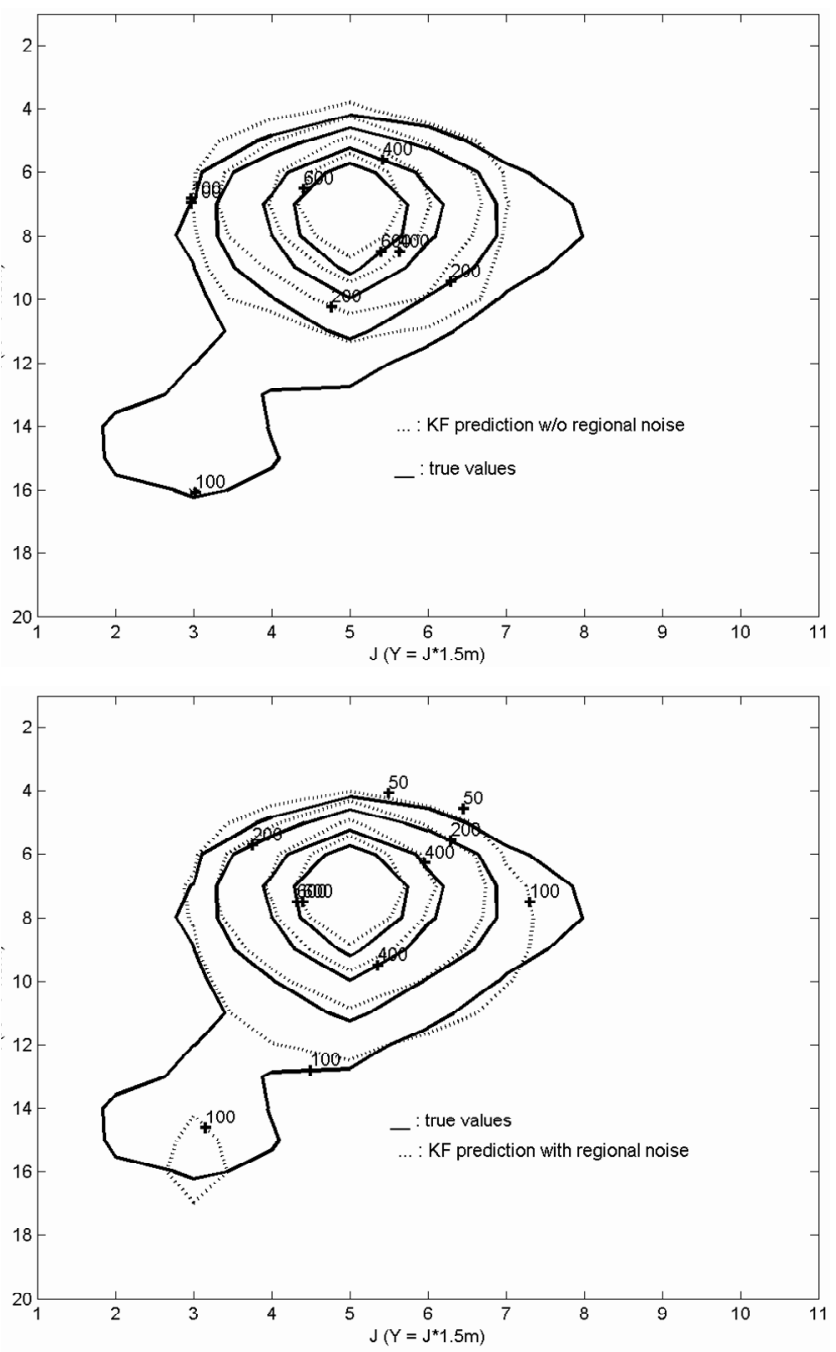

Figure 3. Prediction improved by (a) KF data assimilation with SIG covariance R and (b) data assimilation with regional covariance $\mathrm{R}$ in Case 1 .

order to obtain more comparable results, the KF (with regional error covariance matrix $\mathrm{R}$ ) data assimilation scheme is tested using the same settings for the non-KF and KF with SIG R. 
The prediction from the numerical model that has no data assimilation scheme is shown in dash line in Figure 2. Note that the pollutant plume in the prediction field moves behind the plume of the true field due to the inaccurate V, in downward direction, used in prediction model.

Both Figures $3 \mathrm{a}$ and Figure $3 \mathrm{~b}$ give KF filtered results of pollutant plume at 6.4 day. With the data assimilation scheme, the qualities of model predictions are improved. However, by comparing the two filtered results, the KF using regional noise structure (Figure $3 \mathrm{~b}$ ) is able to follow the irregular shapes of true plume more accurately due to a more effective data assimilation process. In Figure $3 b$, the prediction contours in the significant pollution areas (with pollutant concentration greater than $100 \mathrm{ppm}$ ) follow the true contours more closely than what has shown in Figure $3 \mathrm{a}$ (KF using SIG noise structure).

For quantitatively comparing the results from the models with and without the KF, the Root Mean Squared Error (RMSE) was chosen as the error parameter. $\operatorname{RMSE}(k)$ is defined as:

$$
\operatorname{RMSE}(k)=\sqrt{\frac{1}{N_{i j}-1} \sum_{i, j}\left[C^{E}(i, j, k)-C(i, j, k)\right]^{2}}
$$

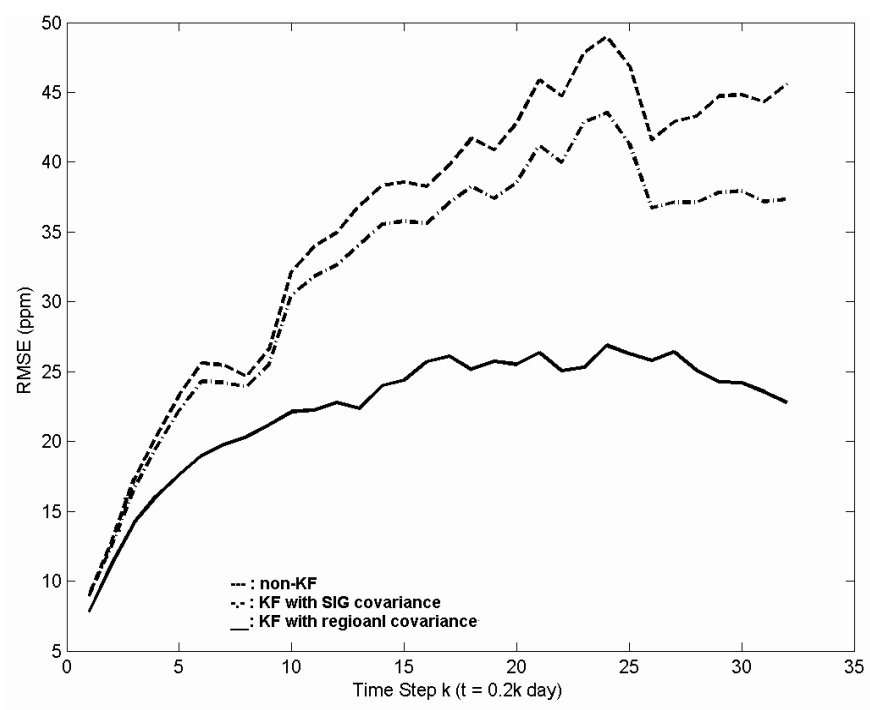

Figure 4. Prediction error (RMSE) for data assimilation with regional noise covariance $\mathrm{R}$ in Case 1 .

The correction effect of different KFs can be seen more clearly in Figure 4, in which the comparison of the average prediction errors (RMSE) for the three schemes, the deterministic model scheme without the KF filter, the model with the KF filter, and the KF model with the regional error structure are calculated at different time steps. As shown in Figure 4, by employing the regional noise KF data assimilation scheme, RMSE, an average deviation to the true stochastic system, is reduced to $20 \mathrm{ppm}$ at 6.4 day. This is a $60 \%$ decrease of RMSE compared to the estimate from the model without KF and about $25 \%$ less compared to the model with KF using SIG noise structure. This correction process of KF involved in pre- diction is very effective in correcting the error that is caused by inaccurate hydrologic parameters. Even without knowing which parameter is inaccurate, this correction process occurred by sampling from only nine sites and applying appropriate observation error covariance matrix $\mathrm{R}$ in $\mathrm{KF}$. As a comparison, Figure 4 suggests that the stochastic model with KF data assimilation is more accurate than the non-KF deterministic model; moreover, the accuracy can be greatly improved by selecting the correct noise statistic structure in the KF scheme.

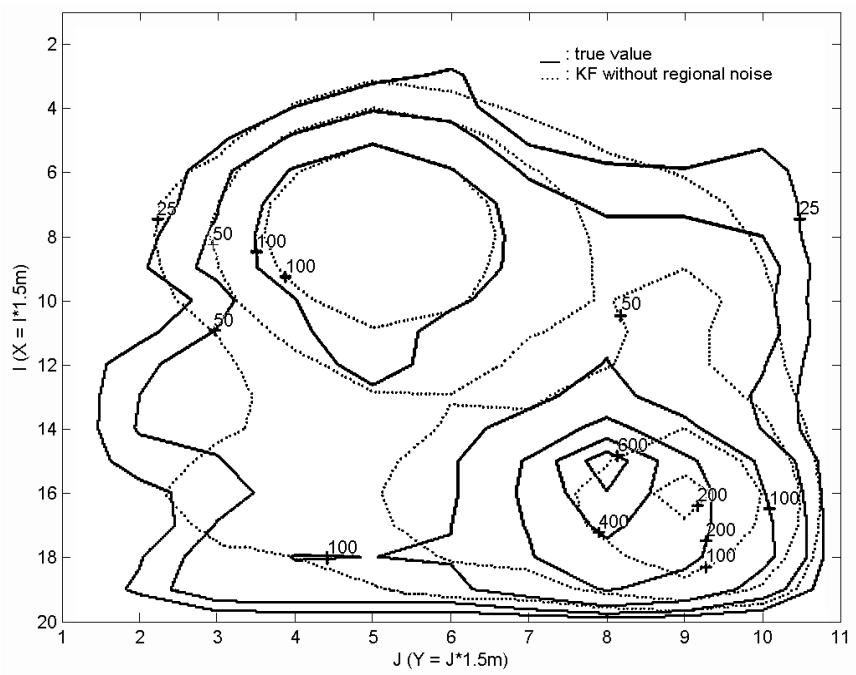

Figure 5. Prediction improved by KF data assimilation (in Case 2: Unknown hidden source).

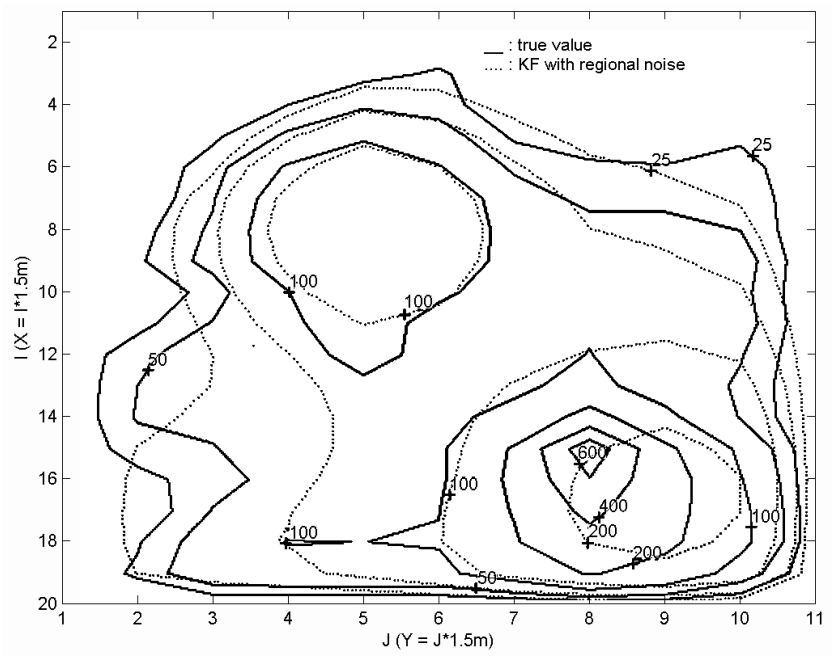

Figure 6. True values and predictions by data assimilation with regional covariance $\mathrm{R}$ (in Case 2).

\subsection{Test Case 2 - Data Assimilation to Reveal Unknown Release Source}

In order to further examine the impact of using a regional noise structure in KF data assimilation scheme, another experiment was conducted using the same transport model with the same level of system/observation errors but with a 

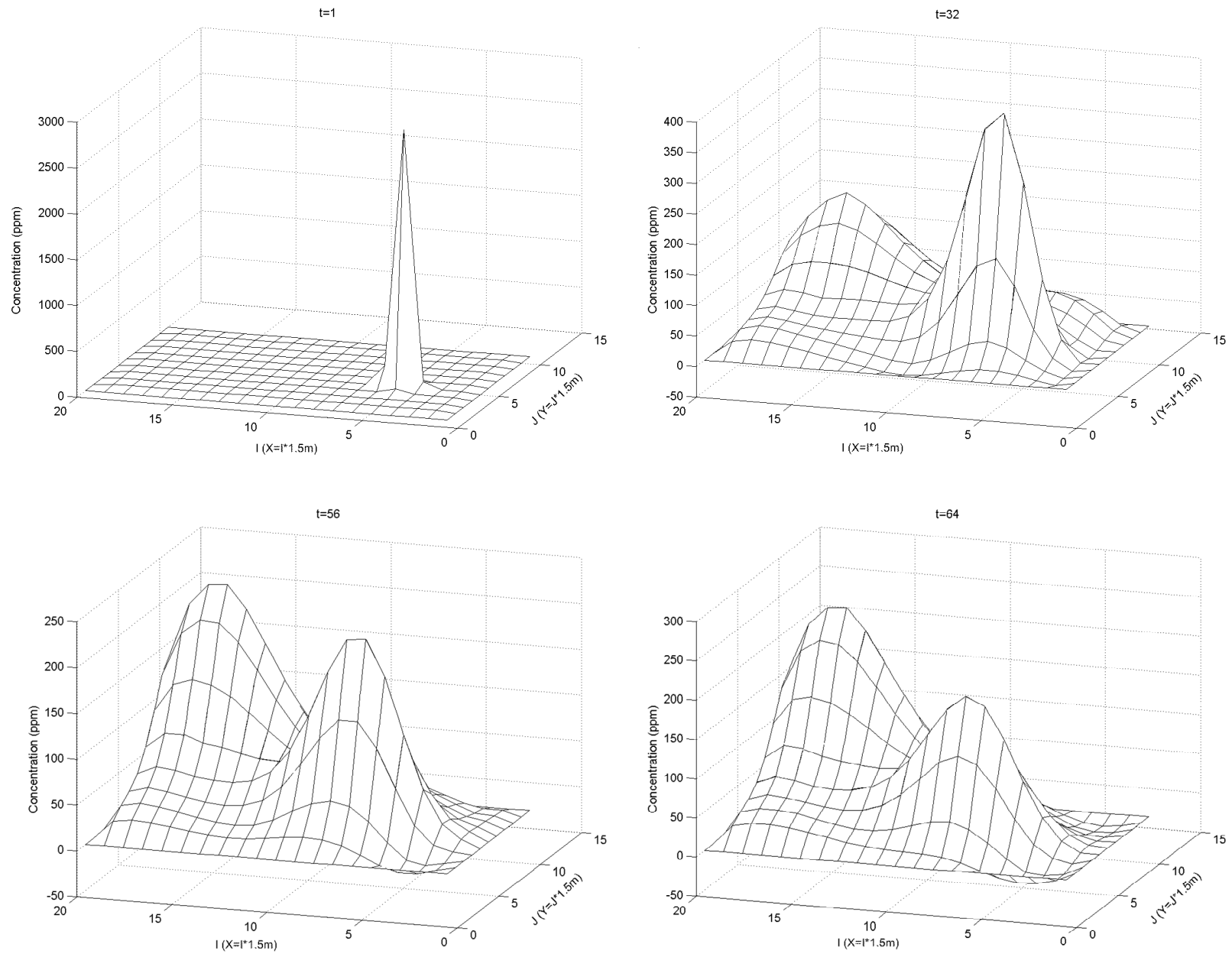

Figure 7. Predictions by data assimilation with regional covariance R (in Case 2).

hidden contaminant release source. In addition to a spontaneous release of $3200 \mathrm{ppm}$, a constant contaminant release source, $800 \mathrm{ppm}$ at gridpoint $(15,8)$, which is designed as an unknown source to the hydrodynamic model and $\mathrm{KF}$, is added to the true field.

Three different prediction models, deterministic hydrodynamic prediction without KF, KF using SIG, and KF using regional noise covariance matrix are examined in this test case. KF filtering results (using SIG and using regional noise) can be seen in Figures 5 and 6, respectively. As expected, the predicttion with $\mathrm{KF}$ using regional noise covariance matrix gives a better result than that with KF using SIG R (Figures 5 and 6).

The revealing process for the hidden source is serialized in Figure 7. By design, the hydrodynamics model and KF are operated without any information about the hidden source. The KF correction process depends only on the very limited indirect information through nine observation sites. At $t=1$, a given contaminant injection is shown as the only release source in the estimated field. As more observation data are assimilated by the regional $\mathrm{KF}$, the hidden source can be seen from the area with growing concentration at $t=32$. At $t=56$, the hidden source has been clearly demonstrated by the data assimilation process. The location and the magnitude of the hidden source in the estimate field that are not seen within the initial simulated period appears at this stage and that this inverse process will eventually show the true field. Figure 8 gives the RMSE for the three prediction models described above. Again, the prediction with KF correction using regional $\mathrm{R}$ is the best of the three. At $t=64$, the RMSE of KF with regional $\mathrm{R}$ is about $70 \%$ lower than the prediction without $\mathrm{KF}$ correction, and about 30\% lower than prediction with $\mathrm{KF}$ using SIG R.

\section{Conclusions}

A two-dimensional advection-dispersion-adsorption subsurface transport model is described and constructed to predict the evolution of contaminant plume. A regional random noise simulation scheme was designed to create "observation" data and system noise. The effectiveness of the Kalman filter (KF) scheme with regional noise covariance matrix was demonstrated by two typical data assimilation applications. Test case 1 has an inaccurate hydrologic parameter and test case 2 has a hidden contaminant release source. 


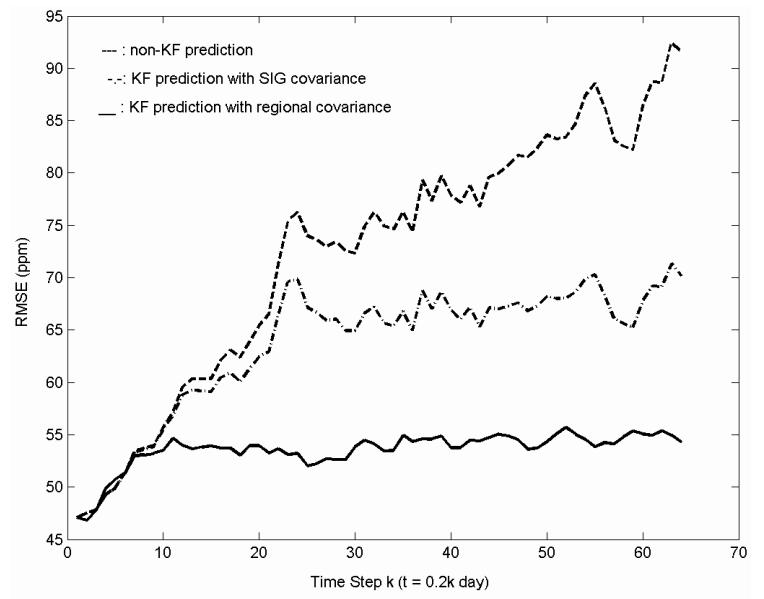

Figure 8. RMSE for data assimilation with regional noise covariance $\mathrm{R}$ (in Case 2).

The KF scheme successfully identifies the correct hydrologic parameters and hidden contaminant sources. The results indicate that the prediction error (RMSE) of the KF scheme using a regional noise covariance matrix is much smaller than that from the model's prediction without KF or that from the KF using a SIG noise matrix. The results from test cases also show the potential applications of a regional noise structure in environmental transport modeling. Applying a regional noise structure in $\mathrm{KF}$ data assimilation improved the KF correction process for inaccurate hydrologic parameters. Furthermore, it improved the inversing process in revealing the location and magnitude of a hidden contaminant source.

Acknowledgments. This work was sponsored by the Department of Energy's Samuel Massie Chair of Excellence Program under grant number DE-FG01-94EW11425. The views and conclusions contained herein are those of the authors and should not be interpreted as necessarily representing the official policies or endorsements, either expressed or implied, of the funding agencies.

\section{References}

Bowles, D.S., and Greney, W.J. (1978). Steady state river quality modeling by sequential extended Kalman filters, Water Resour. Res., 12, 3281-3291.

Brooker, P.I. (2001). Modelling spatial variability using soil profiles in the Riverland of South Australia, Environ. Int., 27(2), 121-126, doi:10.1016/S0160-4120(01)00071-X.

Chang, S.Y., and Jin, A. (2005). Kalman Filtering with Regional Noise to Improve Accuracy of Contaminant Transport Models, J. Environ. Eng., 131(6), 971-982, doi:10.1061/(ASCE)0733-9372(2
005)131:6(971).

Conwell, P.M., Silliman, S.E. and Zheng, L. (1997). Design of a piezometer network for estimation of the variogram of the hydraulic gradient: The role of the instrument, Water Resour. Res., 33(11), 2489-2492.

Ferraresi, M. and Marinelli, A. (1996). An extended formulation of the integrated finite difference method for groundwater flow and transport, J. Hydrol., 175(1-4), 453-471, doi:10.1016/S0022-1694 (96)80020-5

Ferreyra, R.A., Apezteguia, H.P., Sereno, R. and Jones, J.W. (2002). Reduction of soil sampling density using scaled semivariograms and simulated annealing, Geoderma, 110(3-4), 265-289, doi:10.10 16/S0016-7061(02)00234-3.

Ghil, M., Cohn, S.E., Tavantzis, J., Bube, K. and Isaacson, E. (1981). Applications of estimation theory to numerical weather prediction, Dynamic Meteorology: Data Assimilation Methods, L. Bengtsson, M. Ghil, and E. Kallen, Eds., SpringerVerlag, New York, N.Y., pp. 139-224.

Goovaerts, P. (1999). Geostatistics in soil science: state-of-the-art and perspectives, Geoderma, 89(1-2), 1-45, doi:10.1016/S0016-7061(9 8)00078-0.

Gustafsson, N. (1981). A review of method for objective analysis. Dynamic Meteorology: Data Assimilation Methods, L. Bengtsson, M. Ghil, and E. Kallen, Eds., SpringerVerlag, New York, N.Y., pp.17-76.

Heuvelink, G.B.M. and Webster, R. (2001). Modelling soil variation: past, present, and future, Geoderma, 100(3-4), 269-301, doi:10.101 6/S0016-7061(01)00025-8

Harrouni, K.E., Ouazar, D., Wrobel, L.C. and Cheng, A.H.D. (1997). Aquifer parameter estimation by extended Kalman filtering and boundary elements, Engineering Analysis with Boundary Elements, 19(3), 231-237, doi:10.1016/S0955-7997(97)00008-8.

Houtekamer, P. L. and Michell, H. L. (1998). Data assimilation using an ensemble Kalman filter technique. Mon. Weather. Rev., 126, 796-811.

Kalman, R.E., and Bucy, R.S. (1961). New results in linear filtering and prediction theory, Trans. ASME, Ser. D, J. Basic Eng., 83, 95108.

Porter, D., Bruce, G., Jones, W., Huyakorn, P., Hamm, L. and Flach, G. (2000). Data fusion modeling for groundwater systems, J. Contam. Hydrol., 42(2), 303-335, doi:10.1016/S0169-7722(99)00081-9.

Van Geer, F.C. (1982). An equation based theoretical approach to network design for groundwater levels using Kalman filters, Int. Assoc. Hydrol. Sci., Publ., 136, 241-250.

Webster, R., and Oliver, M.A. (1992). Sample adequately to estimate variograms for soil properties, Eur. J. Soil Sci., 43(1), 177-192, doi:10.1111/j.1365-2389.1992.tb00128.x.

Yangxiao, Z., Te Stroet, C.B.M. and Van Geer, F.C. (1991). Using Kalman filtering to improve and quantify the uncertainty of numerical groundwater simulations: 2. application to monitoring network design, Water Resour. Res., 27(8), 1995-2006.

Yu, Y.S., Heidari, M. and Guang-Te, W. (1989). Optimal estimation of contaminant transport in ground water, Water Resources Bulletin, 25(2), 295-300. 